\title{
Perturbation of IGF2BP1 Transcriptome upon the Interplay between miR-486-5p and let-7a
}

\author{
Mourad HA ${ }^{1}$, Assal RA ${ }^{1}$, Youness RA ${ }^{2}$, El Tayebi HM ${ }^{1}$ and Abdelaziz AI ${ }^{3 *}$ \\ ${ }^{1}$ Department Of Pharmacology And Toxicology, German University In Cairo, New Cairo City, Main Entrance Al \\ Tagamoa Al Khames, 11835, Cairo, Egypt \\ ${ }^{2}$ Department of Pharmaceutical Biology, German University in Cairo, New Cairo City, Main Entrance Al Tagamoa Al \\ Khames, 11835, Cairo, Egypt \\ ${ }^{3}$ School of Medicine, NewGiza University, Cairo-Alex Road, 12566, Cairo, Egypt
}

Received: October 28, 2016; Accepted: January 4, 2017; Published: April 1, 2017

*Corresponding author: Ahmed Ihab Abdelaziz, Medicine School, Newgiza University, KM 22 Cairo-Alex Road, 12566, Cairo, Egypt; Tel: +201001488895; E-mail: Ahmed.ihab.abdelaziz@gmail.com

\begin{abstract}
Background: Activation of IGF-1/IGF-1R signaling cascade is a hallmark in Hepatocellular carcinoma (HCC). In our previous work, we showed that miR-486-5p acts as a tumor suppressor miRNA in HCC mainly by vertically blocking IGF-1/IGF-1R axis and its downstream signaling mediators STAT3, mTOR and c-Myc. Recently, it was reported that the proto-oncogene c-Myc directly down-regulates the tumor suppressor miRNA, let-7a, especially in HCC and that let-7 directly targets the oncogenic RNA binding protein IGF2BP1.
\end{abstract}

Aim: Therefore, the main aim of this study was to investigate the indirect interplay between microRNAs; miR-486-5p and miR-let7a through c-MYC thereby its effect on a vital member of IGF-axis, IGF2BP1, in HCC

Methods: Huh-7 cell lines were cultured and transfected using miR-486-5p mimics using lipofection technique. Forty-eight hours post transfection, total RNA was extracted, reverse transcribed into cDNA, and finally amplified and quantified using q-RT-PCR. Impact of miR-486-5p on cell cycle was assessed using cell cycle vectors carrying response elements for the cell cycle protein c-Myc.

Results: Efficient delivery of miR-486-5p in Huh-7 cells was obtained, where mimicked cells showed more than 8000 folds increase in miR-486-5p expression level. Ectopic expression of miR486-5p in Huh-7 cells resulted in a significant decrease in c-Myc protein expression, an increase in the expression level of the tumor suppressor, let-7a and finally forcing the expression of miR-486-5p showed a significant repression of the oncogenic validated target of let-7a, IGF2BP1.

Conclusions: This study shows a novel mechanism of action of the tumor suppressor miR-486-5p. MiR-486-5p was found to indirectly repress an essential member of IGF-axis, the oncogenic RNA binding protein IGF2BP1, mainly through decreasing c-MYC expression and up regulating let-7a expression.

Keywords: Mir-486-5p; Let-7a; IGF2BP2; C-Myc; HCC

\section{Introduction}

Insulin-like growth factor-2 mRNA-binding proteins 1, 2, and 3 (IGF2BP1, IGF2BP2, IGF2BP3) are a family of cytoplasmic mRNA-binding proteins that are essential for development. Normally IGF2BP1 and IGF2BP3 were found to be highly expressed during embryogenesis, whereas they were reported in negligible levels during adulthood, with the exception of reproductive tissues [1]. On the other hand IGF2BP2 is suggested to be normally expressed in adult tissues and appears to have metabolic control and a role in type 2 diabetes and in liver metabolism via directing IGF2 mRNA fate [2-4].

IGF2BPs were found to be re-expressed (de novo synthesized) in several malignancies, including Hepatocellular carcinoma (HCC), where IGF2BP1 and IGF2BP3 were classified as oncofetal cytoplasmic mRNA-binding proteins that appear to have a pivotal role in cancer development and progression. Nonetheless, IGF2BPs expression level was directly correlated with tumor invasion, early recurrence, and poor prognosis of HCC patients [4-6]. Moreover, IGF2BPs were also reported to promote liver steatosis, non-alcoholic fatty liver diseases (NAFLD) which are considered as illustrious risk factors for HCC [7-9].

IGF2BP1 was also reported to inhibit IGF2 mRNA translation in-vitro [10] and in contrast it was shown to enhance IGF2 mRNA levels in mice [11]. On the other hand IGF2BP2 and IGF2BP3 were reported to enhance IGF2 mRNA translation [12]. Our research group has previously shown that the knockdown of the three oncogenic IGF2BPs (IGF2BP1, IGF2BP2, IGF2BP3) by miR-1275 and let-7i, resulted in marked reduction in Huh-7 cells proliferation, migration, cellular viability and clonogenicity properties [13, 14]. Moreover, specific knockdown of IGF2BP1 in particular in multiple liver cancer cell lines resulted in a noticeable reduction in their proliferation and induces apoptosis in these cells. This was attributed to its ability to stabilize the c-MYC and MKI67 mRNAs and increase their protein expression levels [15].On the other hand c-Myc was shown to be an upstream regulator to many miRNAs and validated as a repressor for let-7a [16], which highlights a potential feedback loop between c-Myc, Let-7a and IGF2BP1.

Recently we have shown that miR-486-5p acts as tumor 
suppressor miRNA through directly targeting IGF-1, IGF-1R and its downstream signaling mediators c-Myc, mTOR, STAT3 in Huh7 cells $[17,18]$.Thus hypothetically miR-486-5p may regulate IGF2BP1 through the manipulation of the expression of c-MYCLet-7a axis, so the main aim of this study was to investigate the interplay between c-Myc and Let-7a via miR-486-5p and its impact on IGF2BP1.

\section{Materials and Methods}

\section{Cell culture and oligonucleotide transfection}

Huh-7 cells were cultured in DMEM (Dulbecco's Modified Eagle Medium) supplemented with 10\% FBS (Fetal Bovine Serum), 1\% penicillin/streptomycin/mycozap (Lonza, Switzerland). Cells were transfected with mimics of miR-486$5 p$ (Qiagen, Germany) using HiPerFect Transfection Reagent (Qiagen, Germany), according to the manufacturer's protocol. Mock cells are cells exposed to transfection reagent only.

\section{MicroRNA and mRNA extraction and quantification HuH-7 cell line}

Total mRNA and microRNAs were extracted from Huh-7 cells using BIOZOL RNA Extraction Reagent (Bioer Technology, China). Complementary DNA was generated using reverse transcription (Applied Biosystems, USA). Relative expression of miR-486-5p was normalized to RNU6B in each sample, while IGF2BP1 relative expression was normalized to beta-2 microglobulin (B2M), and quantified using TaqMan Realtime quantitative polymerase chain reaction (RTqPCR) (Applied Biosystems). Relative expression was calculated using the $2-\Delta \Delta \mathrm{CT}$ method.

\section{Reporter constructs and Luciferase assay}

Huh-7 cells were transfected with a vector containing the enhancer element of cell cycle regulatory proteins, c-Myc, and containing an unspecific binding site as p-Luc vector (Clontech, Germany). Huh-7 cells were transfected with $2 \mu \mathrm{g}$ of the constructs using SuperFect (Qiagen) then co-transfected $24 \mathrm{~h}$ later with miR-486-5p mimics using HiPerFect (Qiagen). Relative luciferase activity was measured after $48 \mathrm{~h}$ by the Luciferase Reporter Assay Kit (Biovision, USA). It was measured at $615 \mathrm{~nm}$ using Wallac 1420 Victor2 Multilabel Counter (Perkin Elmer, USA)

\section{Statistical analysis}

All experiments were performed in triplicates and repeated at least three times. The data were expressed as the mean \pm standard error of the mean (SEM). All analyses, unless otherwise stated, were performed using GraphPad Prism version 5 and a two-tailed value of $\mathrm{P}<0.05$ was considered statistically significant with Student's t-test.

\section{Result}

\section{Transfection Efficiency of miR-486-5p oligonucleotides}

For the purpose of assessing the impact of miR-4865p Huh-7 cell line, miR-486-5p mimics were transfected into cultured Huh-7 cells. In order to confirm efficient transfection, miR-486-5p was quantified $48 \mathrm{~h}$ post-transfection using qRTPCR, where a dramatic up-regulation of miR-486-5p of about 8000 folds was observed in mimicked cells compared to mock untransfected cells $(\mathrm{P}=0.0040)$ as shown in Figure 1.

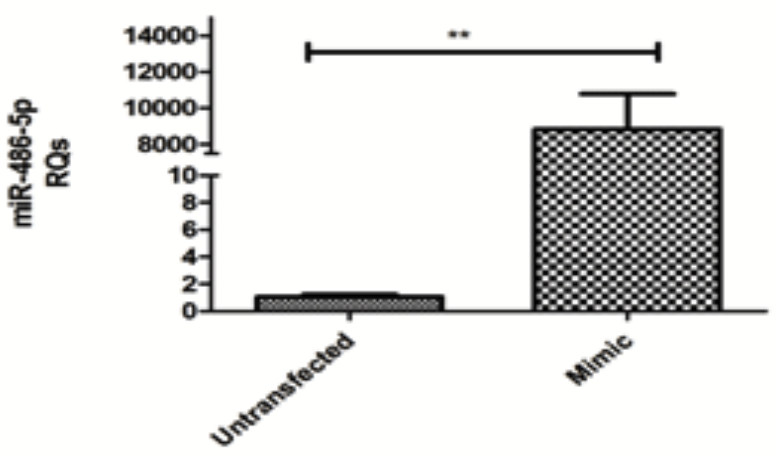

Figure 1: Transfection efficiency of miR-486-5p in Huh7 cells. Mir-486-5p Expression Level Was Assessed Using Qrt-PCR And RNU6B Was Used As An Internal Control. Mir-486-5p Showed More Than 8000 Folds Increase In Mimicked Huh-7 Cells Compared To Mock Untransfected Cells

\section{Impact of miR-486-5p on cell cycle progression through c-MYC expression}

Mimicked miR-486-5p cells showed a significant $(\mathrm{P}=0.0456)$ down-regulation of c-MYC expression compared to mock untransfected cells using luciferase reported assay as shown in Figure 2

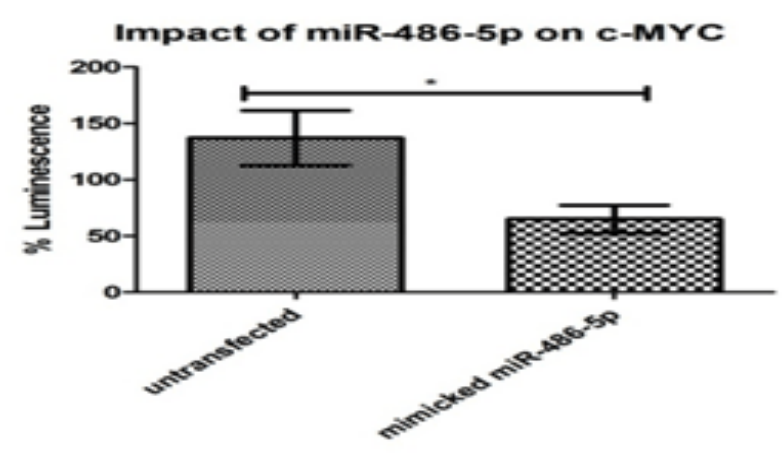

Figure 2: Impact of mi-486-5p on c-Myc protein level Mir-486-5p Mimics Led To A Significant Decrease In C-Myc Luciferase Activity Compared To Mock Untransfected Cells In Huh-7 Cells Transfected With Pmyc-TA-Luc Vector. 


\section{Impact of miR-486-5p mimicked cells on Let-7a expression levels}

Mimicking of miR-486-5p expression showed a significant up-regulation of let-7a expression in the Huh-7 cells $(\mathrm{P}=0.0471)$ compared to the mock untransfected cells as shown in Figure 3.

The impact of miR-486 on miR-let-7a expression in Huh-7 cells

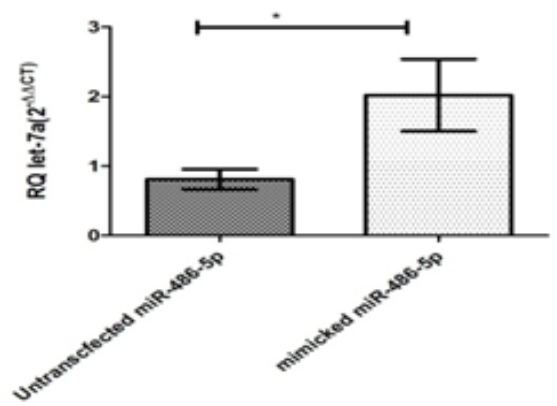

Figure 3: Impact of miR-486-5p on let-7a expression level in Huh7 cells Let-7a expression level was assessed using qRT-PCR and RNU6B was used as an internal control. Let-7a expression level was significantly augmented in mimicked Huh-7 cells compared to mock untransfected cells.

\section{Impact of miR-486-5p on IGF2BP1 expression in Huh-7 cells}

The impact of miR-486-5p manipulation was assessed using qRT-PCR, where mimicking of miR-486-5p in Huh-7 cells resulted in a significant down-regulation of IGF2BP1 mRNA levels $(\mathrm{P}=0.0044)$ compared to mock untransfected cells. However, Anti-miR-486-5p showed non-significant difference in IGF2BP1 expression levels compared to mock untransfected cells as shown in Figure 4.

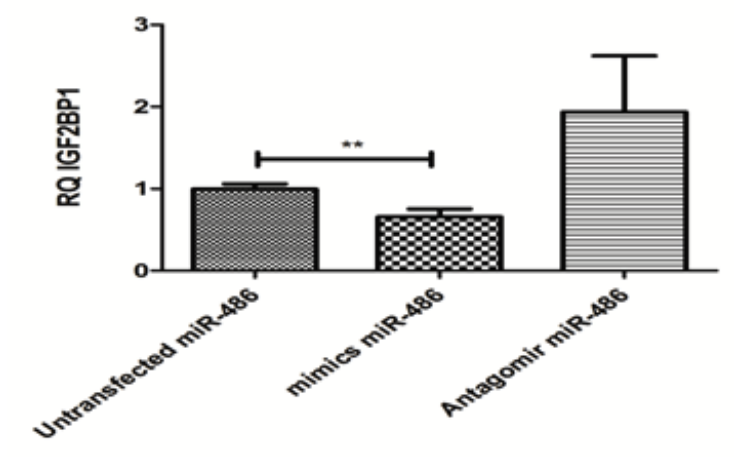

Figure 4: The mRNA level of IGF2BP1 was assessed using qPT-PCR and normalized to B2M as an internal control. Ectopic expression of miR486-5p resulted in an increase in IGF2BP1 mRNA level in mimicked Huh7 cells compared to mock untransfected cells. While antagomirs showed no effect when compared to mock untransfected cells

\section{Discussion}

Recently we have shown that miR-486-5p could act as a tumor suppressor miRNA upon forcing its expression in HCC mainly through decreasing IGF-1/IGF-1R axis and its downstream mediators mTOR, STAT3, c-MYC [17]. On the other hand previous studies have shown that c-MYC regulates the expression of let-7a [16], which in turn regulates IGF2BP1[19, 20].

This study aimed at further characterizing the mechanistic role for miR-486-5p by examining its indirect impact on IGF2BP1 through c-MYC and let-7a loop. Loss and gain of function experiments for miR-486-5p in Huh-7 cells repressed the expression of c-MYC on the protein level, which supports previous findings where c-MYC expression was manipulated by miRNAs like miR-135b and miR-181a [21].Then the impact on let-7a via c-MYC was investigated through intended over expression of miR-486-5p in Huh-7 cells compared to the mockuntransfected cells, forced expression of miR-486-5p resulted in a significant up-regulation of let-7a in Huh-7 cells. This goes along with the idea that repressing c-MYC relives let-7a from its inhibitory effect.

The impact of miR-486-5p on IGF2BP1was then investigated expression through forcing its expression in Huh7 cells, which resulted in a significant down regulation of IGF2BP1 mRNA. This might show indirect impact of miR-486$5 p$ on IGF2BP1 through c-MYC and let-7a loop. This goes in line with another study where we have shown that c-MYC as an intermediate player between E2f and miR-17-5p creating a triad of E2F/c-MYC/miR-17-5p in Systemic lupus erythematosus (SLE) [22]. This also simulates another study where c-MYC was able to regulate CCDN2 through manipulating the expression of miRNAs let-7a, miR-16 and miR-29b in Ewing's sarcoma [16].

In conclusion This study showed that miR-486-5p interplays with let-7a through repressing c-MYC, which is an upstream regulator of let-7a hence relieving the inhibitory effect of c-MYC on let-7a enhancing its expression and increasing the

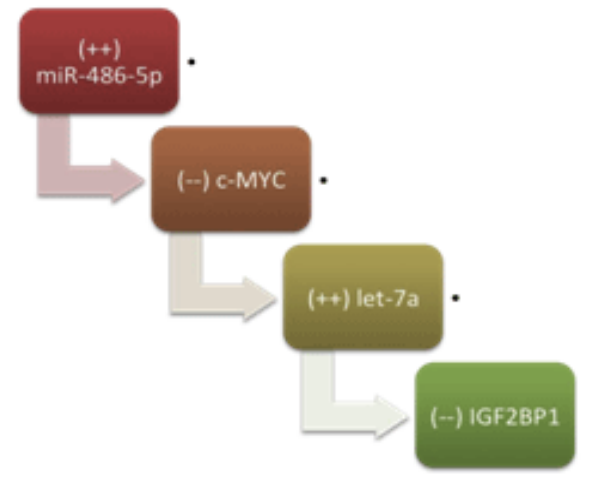

Figure 5: Schematic Representation of Interplay between miR-486-5p and let-7a in Huh-7 cells 


\section{References}

1. Hammer NA, Hansen TV, Byskov AG, Rajpert-De Meyts E, Grøndahl ML, Bredkjaer HE, et al. Expression of IGF-II mRNA-binding proteins (IMPs) in gonads and testicular cancer. Reproduction. 2005;130(2):203-212. doi:10.1530/ rep.1.00664

2. Le HT, Sorrell AM, Siddle K. Two isoforms of the mRNA binding protein IGF2BP2 are generated by alternative translational initiation. PLoS One. 2012;7(3):e33140. doi:10.1371/journal. pone. 0033140

3. Yisraeli JK. VICKZ proteins: a multi-talented family of regulatory RNA-binding proteins. Biol Cell. 2005;97(1):87-96. doi:10.1042/BC20040151

4. Yaniv K, Yisraeli JK. The involvement of a conserved family of RNA binding proteins in embryonic development and carcinogenesis. Gene. 2002;287(1-2):49-54.

5. Wachter DL, Kristiansen G, Soll C, Hellerbrand C, Breuhahn K, Fritzsche $\mathrm{F}$, et al. Insulin-like growth factor II mRNA-binding protein 3 (IMP3) expression in hepatocellular carcinoma. A clinicopathological analysis with emphasis on diagnostic value. Histopathology. 2012;60(2):278-286. doi:10.1111/ j.1365-2559.2011.04091.x

6. Jeng YM, Chang CC, Hu FC, Chou HY, Kao HL, Wang TH, et al. RNA-binding protein insulin-like growth factor II mRNAbinding protein 3 expression promotes tumor invasion and predicts early recurrence and poor prognosis in hepatocellular carcinoma. Hepatology. 2008;48(4):1118-1127. doi:10.1002/ hep.22459

7. Laggai S, Kessler SM , Boettcher S , Lebrun V , Gemperlein $K$, et al. The IGF2 mRNA binding protein p62/IGF2BP2-2 induces fatty acid elongation as a critical feature of steatosis. J Lipid Res. 2014;55(6):1087-1097. doi:10.1194/jlr.M045500

8. Kessler SM , Pokorny J, Zimmer V, Laggai S, Lammert F, Bohle RM, et al. IGF2 mRNA binding protein p62/IMP2-2 in hepatocellular carcinoma: antiapoptotic action is independent of IGF2/PI3K signaling. Am J Physiol Gastrointest Liver Physiol. 2013;304(4):328-336. doi:10.1152/ajpgi.00005.2012

9. Maolong Lu, Nakamura RM, Dent ED, Zhang JY, Nielsen FC, Christiansen J, et al. Aberrant expression of fetal RNA-binding protein p62 in liver cancer and liver cirrhosis. Am J Pathol. 2001;159(3):945-953. doi:10.1016/S0002-9440(10)61770-1

10. Nielsen J, Christiansen J, Lykke-Andersen J, Johnsen AH, Wewer UM, Nielsen FC. A family of insulin-like growth factor II mRNAbinding proteins represses translation in late development. Mol Cell Biol. 1999;19(2):1262-1270.

11. Tessier CR, Doyle GA, Clark BA, Pitot HC, Ross J . Mammary tumor induction in transgenic mice expressing an RNAbinding protein. Cancer Res. 2004;64(1):209-214.

12. Dai N, Rapley J, Angel M, Yanik MF, Blower MD, Avruch J. mTOR phosphorylates IMP2 to promote IGF2 mRNA translation by internal ribosomal entry. Genes Dev. 2011;25(11):1159-1172. doi:10.1101/gad.2042311

13. Fawzy IO, Hamza MT, Hosny KA, Esmat G, Abdelaziz AI. Abrogating the interplay between IGF2BP1, 2 and 3 and IGF1R by let-7i arrests hepatocellular carcinoma growth. Growth Factors. 2016;34(1-2):42-50. doi:10.3109/08977194.2016.1 169532

14. Fawzy IO, Hamza MT, Hosny KA, Esmat G, El Tayebi HM, Abdelaziz AI. miR-1275: A single microRNA that targets the three IGF2-mRNA-binding proteins hindering tumor growth in hepatocellular carcinoma. FEBS Lett. 2015;589(17):22572265. doi:10.1016/j.febslet.2015.06.038

15. Noubissi FK, Nikiforov MA, Colburn N, Spiegelman VS. Transcriptional Regulation of CRD-BP by c-myc: Implications for c-myc Functions. Genes Cancer. 2010;1(10):1074-1082. doi:10.1177/1947601910395581

16. Kawano M, Tanaka K, Itonaga I, Iwasaki T, Tsumura H. c-Myc Represses Tumor-Suppressive microRNAs, let-7a, miR-16 and miR-29b, and Induces Cyclin D2-Mediated Cell Proliferation in Ewing's Sarcoma Cell Line. PLoS One. 2015;10(9):e0138560. doi:10.1371/journal.pone.0138560

17. Youness RA, Tayebi HMEI, Assal RA, Hosny KA, Esmat G, Abdelaziz AI. MicroRNA-486-5p enhances HCC tumor suppression through repressing IGF-1R and its downstream mTOR, STAT3 and c-Myc. Oncol Lett. 2016;12(4):2567-2573. doi:10.3892/ol.2016.4914

18. Youness RA, Rahmoon MA , Assal RA , Gomaa AI , Hamza MT , Waked I, et al. Contradicting interplay between insulinlike growth factor- 1 and miR-486-5p in primary NK cells and hepatoma cell lines with a contemporary inhibitory impact on HCC tumor progression. Growth Factors. 2016;34(3-4):128140. doi: 10.1080/08977194.2016.1200571

19. Chen W, Yan ZH, Wang YM, Xu BY, Deng GH . Genome-wide microarray-based analysis of miRNAs expression in patients with acute-on-chronic liver failure. Hepatobiliary Pancreat Dis Int. 2014;13(1):32-39.

20. Cheng M, Si Y, Niu Y, Liu X, Li X, Zhao J, et al. High-throughput profiling of alpha interferon- and interleukin-28B-regulated microRNAs and identification of let-7s with anti-hepatitis $\mathrm{C}$ virus activity by targeting IGF2BP1. J Virol. 2013;87(17):97079718. doi:10.1128/JVI.00802-13

21. Liu Z, Zhang G, Li J, Liu J, Lv P. The tumor-suppressive microRNA-135b targets c-myc in osteoscarcoma. PLoS One. 2014;9(7):e102621. doi:10.1371/journal.pone.0102621

22. Sarhan RA, Aboelenein HR, Sourour SK, Fawzy IO, Salah S, Abdelaziz AI. Targeting E2F1 and c-Myc expression by microRNA-17-5p represses interferon-stimulated gene MxA in peripheral blood mononuclear cells of pediatric systemic lupus erythematosus patients. Discov Med. 2015;19(107):419-425. 